TRANSACTIONS OF THE

AMERICAN MATHEMATICAL SOCIETY

Volume 355, Number 12, Pages 4767-4779

S 0002-9947(03)03333-6

Article electronically published on July 24, 2003

\title{
NON-TRIVIAL QUADRATIC APPROXIMATIONS TO ZERO OF A FAMILY OF CUBIC PISOT NUMBERS
}

\author{
PETER BORWEIN AND KEVIN G. HARE
}

\begin{abstract}
This paper gives exact rates of quadratic approximations to an infinite class of cubic Pisot numbers. We show that for any cubic Pisot number $q$, with minimal polynomial $p$, such that $p(0)=-1$, and where $p$ has only one real root, then there exists a $C(q)$, explicitly given here, such that:
\end{abstract}

(1) For all $\epsilon>0$, all but finitely many integer quadratics $P$ satisfy

$$
|P(q)| \geq \frac{C(q)-\epsilon}{H(P)^{2}}
$$

where $H$ is the height function.

(2) For all $\epsilon>0$ there exists a sequence of integer quadratics $P_{n}(q)$ such that

$$
\left|P_{n}(q)\right| \leq \frac{C(q)+\epsilon}{H\left(P_{n}\right)^{2}} .
$$

Furthermore, $C(q)<1$ for all $q$ in this class of cubic Pisot numbers. What is surprising about this result is how precise it is, giving exact upper and lower bounds for these approximations.

\section{INTRODUCTION}

The study of continued fractions is the study of finding good rational approximations $\frac{a_{n}}{b_{n}}$ to irrational numbers $\alpha$ [1, 5]. Finding these good rational approximations is equivalent to finding good linear approximations $b_{n} \alpha-a_{n}$ to zero. The idea of finding good linear approximations to zero extends to finding good quadratic approximations of an irrational number $q$ to zero of the form $A_{n} q^{2}+B_{n} q+C_{n}$. Clearly, if $q$ is quadratic, then $A_{n}, B_{n}$ and $C_{n}$ can be solved exactly in the integers. Here we consider the case when $q$ is a particular type of cubic Pisot number. Recall:

Definition 1. A Pisot number is a real algebraic integer, all of whose conjugates are of modulus strictly less than 1. A Pisot polynomial is the minimal polynomial of a Pisot number.

The class of cubic Pisot numbers that we consider is:

Definition 2. Let $\mathcal{P}$ be the set of Pisot numbers with Pisot polynomial $p$ such that:

(1) $p(0)=-1$,

Received by the editors March 1, 2001.

2000 Mathematics Subject Classification. Primary 11Y60, 11Y40.

Key words and phrases. Pisot numbers, continued fraction, quadratic approximation.

The first author was supported by MITACS and by NSERC of Canada.

The research of the second author was supported by MITACS and by NSERC of Canada. 
(2) $\operatorname{deg}(p)=3$,

(3) $p$ has only one real root.

We see that requirement (3) does not necessarily follow from requirements (1) and (2) since $x^{3}-3 x^{2}-4 x-1$ satisfies the first two requirements, yet has three real roots, of approximately $-0.6920214716,-0.3568958679$ and 4.048917340 . We easily see that this class is non-trivial and infinite.

Let us again consider the case of linear approximations to zero as it arises from continued fractions. It is known [1] that if $\alpha$ is an algebraic number and $A$ and $B$ are integers such that $|B \alpha-A| \leq \frac{1}{2 B}$, then $A / B$ is a convergent of $\alpha$. From this it follows that for $0<b<B$ we have $|B \alpha-A|<|a \alpha-b|$. In other words, $P(x)=B x-A$ is the best non-trivial linear approximation to zero at $\alpha$ bounded by height $\max (A, B)$. We prove a similar result here for quadratic approximations of $q \in \mathcal{P}$ to zero.

The classic result, known as Louiville's inequality (see for example [10]), shows that if $\alpha$ is an algebraic number of degree $d \geq 2$, then there exists a $D(\alpha)$ such that

$$
\left|\alpha-\frac{p}{q}\right| \geq \frac{D(\alpha)}{q^{d}}, p, q \in \mathbb{Z}, q \geq 1,
$$

or equivalently,

$$
|q \alpha-p| \geq \frac{D(\alpha)}{q^{d-1}} .
$$

Another result worth noting (see Schmidt [12]) shows that for every $\epsilon>0$, and an algebraic number $q$ where $1, q$ and $q^{2}$ are independent, there are only a finite number of integer quadratics $P$ such that

$$
|P(q)| \leq \frac{1}{H(P)^{2+\epsilon}} .
$$

Here $H$ is the height of a polynomial $P$. What Schmidt shows is actually more general than this, showing that if $1, \alpha$ and $\beta$ are linearly independent algebraic numbers, then there are only a finite number of solutions to

$$
|a \alpha+b \beta+c|<\max (a, b)^{-2-\epsilon}
$$

for integers $a, b$ and $c$.

Roth's classic result [11 states that if $\alpha$ is an algebraic number, then for all $\epsilon>0$,

$$
\left|\alpha-\frac{p}{q}\right| \leq \frac{1}{q^{2+\epsilon}}
$$

has only finitely many solutions over the integers. The main open question with respect to this is whether an effective upper bound for $q$ (dependent on $\epsilon$ ) can be found. This paper answers the equivalent question for quadratic approximations for $q \in \mathcal{P}$, finding an explicit upper bound for the height of $P$, dependent on $\epsilon$, after which there are no solutions to

$$
|P(q)| \leq \frac{1}{H(P)^{2+\epsilon}}
$$

In Section 2 we prove the existence of a sequence of good quadratic approximations to zero for $q \in \mathcal{P}$. Section 3 proves that the sequence described in Section 2 is the best possible. In Section 4 we show that $C(q)<1$ for all $q \in \mathcal{P}$, where $C(q)$ is a factor which indicates how good an approximation the sequence of quadratics gives. Section $[5$ determines a bound on the height of a quadratics $P$ (dependent 
on $\epsilon$ ) after which there are no solutions to $P(q) \leq \frac{1}{H(P)^{2+\epsilon}}$. Finally, Section 6 gives some conclusions and lists some open problems.

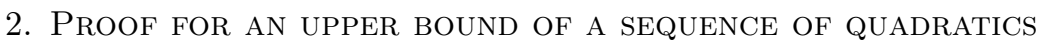

This section shows the existence of a sequence of quadratics that give good approximations to zero when evaluated at $q$. First we need to define $C(q)$. Here and throughout, $\tau$ represents the Golden ratio (the maximal root of $x^{2}-x-1$ ).

Definition 3. Let $q \in \mathcal{P}$, with conjugates $q_{1}$ and $q_{2}$. If $q>\tau$, then

$$
\begin{aligned}
C(q):=\min \left(\frac{q^{4}}{\left(q_{1} q+q+q_{1}\right)\left(q_{2} q+q+q_{2}\right)\left(q-q_{1}\right)\left(q-q_{2}\right)},\right. \\
\left.\frac{q^{4}}{\left(q_{1} q-q-q_{1}\right)\left(q_{2} q-q-q_{2}\right)\left(q-q_{1}\right)\left(q-q_{2}\right)}\right) ;
\end{aligned}
$$

while if $q<\tau$, then

$$
\begin{array}{r}
C(q):=\min \left(\frac{q^{4}}{\left(q_{1} q+q+q_{1}\right)\left(q_{2} q+q+q_{2}\right)\left(q-q_{1}\right)\left(q-q_{2}\right)},\right. \\
\frac{q^{4}}{\left(q_{1} q-q-q_{1}\right)\left(q_{2} q-q-q_{2}\right)\left(q-q_{1}\right)\left(q-q_{2}\right)} \\
\left.\frac{q^{2}}{\left(q_{1} q+1\right)\left(q_{2} q+1\right)\left(q-q_{1}\right)\left(q-q_{2}\right)}\right) .
\end{array}
$$

For convenience we denote

$$
C_{1}(q)=\frac{q^{4}}{\left(q_{1} q+q+q_{1}\right)\left(q_{2} q+q+q_{2}\right)\left(q-q_{1}\right)\left(q-q_{2}\right)}
$$

and

$$
C_{2}(q)=\frac{q^{4}}{\left(q_{1} q-q-q_{1}\right)\left(q_{2} q-q-q_{2}\right)\left(q-q_{1}\right)\left(q-q_{2}\right)}
$$

Theorem 2.1. If $q \in \mathcal{P}$, then for all $\epsilon>0$ there exists a sequence of integer quadratics $P_{n}(x)=A_{n} x^{2}+B_{n} x+C_{n}$ such that

$$
\left|P_{n}(q)\right|=\left|A_{n} q^{2}+B_{n} q+C_{n}\right| \leq \frac{C(q)+\epsilon}{H(P)^{2}} .
$$

Proof. Let $q \in \mathcal{P}$ and let $p$ be the minimal polynomial of $q$. Notice that $p$ is of the form $x^{3}+r_{2} x^{2}+r_{1} x-1$. We notice in $\mathbb{Z}[x] / p(x)$, that $x\left(x^{2}+r_{2} x+r_{1}\right)=1$, or equivalently $\frac{1}{x}$ is in $\mathbb{Z}[x] / p(x)$. So we can write $P_{n}(x)=A_{n} x^{2}+B_{n} x+C_{n}=\frac{1}{x^{n}}$ in the ring $\mathbb{Z}[x] / p(x)$. Then $A_{n}, B_{n}$ and $C_{n}$ can be written as

$$
\begin{aligned}
& A_{n}=a_{1} \beta^{n}+a_{2} \gamma^{n}+a_{3} \bar{\gamma}^{n}, \\
& B_{n}=b_{1} \beta^{n}+b_{2} \gamma^{n}+b_{3} \bar{\gamma}^{n}, \\
& C_{n}=c_{1} \beta^{n}+c_{2} \gamma^{n}+c_{3} \bar{\gamma}^{n}
\end{aligned}
$$

where $\beta=\frac{1}{q}$ with $\gamma$ and $\bar{\gamma}$ the conjugates to $\beta$. It is worth noting that $a_{2}=\bar{a}_{3}$, $b_{2}=\bar{b}_{3}$ and $b_{2}=\bar{b}_{3}$. Since $\beta<1$, for any $\epsilon>0$ there exists an $N$, such that for all $n \geq N$ we have $\left|a_{1} \beta^{n}\right|,\left|b_{1} \beta^{n}\right|,\left|c_{1} \beta^{n}\right|<\epsilon$. (Basically, we can ignore $\beta$ from here on in.) We know that $\gamma$ has an irrational angle in the complex plane, and hence we can get arbitrarily close to any angle with powers of $\gamma$. Thus the height of $P_{n}$ can 
be written as

$$
\begin{aligned}
H\left(P_{n}\right) & =\max \left(\left|A_{n}\right|,\left|B_{n}\right|,\left|C_{n}\right|\right) \\
\leq & \max \left(\left|a_{2} \gamma^{n}+a_{3} \bar{\gamma}^{n}\right|,\left|b_{2} \gamma^{n}+b_{3} \bar{\gamma}^{n}\right|,\left|c_{2} \gamma^{n}+c_{3} \bar{\gamma}^{n}\right|\right)+\epsilon \\
& \leq\left|\gamma^{n}\right| \max \left(\left|a_{2}\left(\frac{\gamma}{|\gamma|}\right)^{n}+a_{3}\left(\frac{\bar{\gamma}}{|\gamma|}\right)^{n}\right|,\left|b_{2}\left(\frac{\gamma}{|\gamma|}\right)^{n}+b_{3}\left(\frac{\bar{\gamma}}{|\gamma|}\right)^{n}\right|,\right. \\
& \left.\left|c_{2}\left(\frac{\gamma}{|\gamma|}\right)^{n}+c_{3}\left(\frac{\bar{\gamma}}{|\gamma|}\right)^{n}\right|\right)+\epsilon \\
\leq & \left|\gamma^{n}\right| \max \left(\left|a_{2} \alpha^{n}+a_{3} \bar{\alpha}^{n}\right|,\left|b_{2} \alpha^{n}+b_{3} \bar{\alpha}^{n}\right|,\left|c_{2} \alpha^{n}+c_{3} \bar{\alpha}^{n}\right|\right)+\epsilon .
\end{aligned}
$$

Here $\alpha$ is an irrational angled complex number with modulus 1 . Thus for any $\epsilon>0$, we can choose a subsequence of $n$, say $n_{i}$, such that

$$
H\left(P_{n_{i}}\right) \leq\left|\gamma^{n_{i}}\right|\left(\min _{|\theta|=1}\left(\max \left(\left|a_{2} \theta+a_{3} \bar{\theta}\right|,\left|b_{2} \theta+b_{3} \bar{\theta}\right|,\left|c_{2} \theta+c_{3} \bar{\theta}\right|\right)\right)+\epsilon\right) .
$$

Let us put $U(q):=\min _{|\theta|=1}\left(\max \left(\left|a_{2} \theta+a_{3} \bar{\theta}\right|,\left|b_{2} \theta+b_{3} \bar{\theta}\right|,\left|c_{2} \theta+c_{3} \bar{\theta}\right|\right)\right)^{2}$. (We later show that $U(q) \leq C(q)$.) On noticing that $|\gamma|^{2}=q$, we get

$$
\begin{aligned}
H\left(P_{n_{i}}\right)^{2} & \leq(U(q)+\epsilon)|\gamma|^{2 n_{i}}, \\
H\left(P_{n_{i}}\right)^{2} & \leq(U(q)+\epsilon) q^{n_{i}}, \\
\frac{1}{q^{n_{i}}} & \leq \frac{U(q)+\epsilon}{H\left(P_{n_{i}}\right)^{2}}, \\
\left|P_{n_{i}}(q)\right| & \leq \frac{U(q)+\epsilon}{H\left(P_{n_{i}}\right)^{2}} .
\end{aligned}
$$

We now show that for $q>\tau$

$$
U(q)=\min _{|\theta|=1}\left(\max \left(\left|a_{2} \theta+a_{3} \bar{\theta}\right|,\left|b_{2} \theta+b_{3} \bar{\theta}\right|,\left|c_{2} \theta+c_{3} \bar{\theta}\right|\right)\right)^{2}
$$

occurs when $\left|b_{2} \theta+b_{3} \bar{\theta}\right|=\left|c_{2} \theta+c_{3} \bar{\theta}\right|$ with $\left|a_{2} \theta+a_{3} \bar{\theta}\right|<\left|b_{2} \theta+b_{3} \bar{\theta}\right|$. We solve for $\theta$ such that $\left|b_{2} \theta+b_{3} \bar{\theta}\right|=\left|c_{2} \theta+c_{3} \bar{\theta}\right|$ and show that $\left|a_{2} \theta+a_{3} \bar{\theta}\right|<\left|b_{2} \theta+b_{3} \bar{\theta}\right|$ is a consequence of this (for $q>\tau$ ). We see that $a_{2} \theta+a_{3} \bar{\theta}, b_{2} \theta+b_{3} \bar{\theta}$ and $c_{2} \theta+c_{3} \bar{\theta}$ are all real. Thus there are two cases to solve for when $\left|b_{2} \theta+b_{3} \bar{\theta}\right|=\left|c_{2} \theta+c_{3} \bar{\theta}\right|$. The first is $b_{2} \theta+b_{3} \bar{\theta}=c_{2} \theta+c_{3} \bar{\theta}$ and the second is $b_{2} \theta+b_{3} \bar{\theta}=-c_{2} \theta-c_{3} \bar{\theta}$.

Before looking at these two cases, we need to explicitly solve for $a_{2}, a_{3}, b_{2}, b_{3}$, $c_{2}$ and $c_{3}$ in equations (1), (2) and (3). This gives

$$
\begin{aligned}
& a_{2}=\frac{-1}{\left(q_{1}-q_{2}\right)\left(q-q_{1}\right)}, \\
& a_{3}=\frac{1}{\left(q_{1}-q_{2}\right)\left(q-q_{2}\right)}, \\
& b_{2}=\frac{\left(q+q_{2}\right)}{\left(q_{1}-q_{2}\right)\left(q-q_{1}\right)}, \\
& b_{3}=\frac{-\left(q+q_{1}\right)}{\left(q_{1}-q_{2}\right)\left(q-q_{2}\right)}, \\
& c_{2}=\frac{-q q_{2}}{\left(q_{1}-q_{2}\right)\left(q-q_{1}\right)}, \\
& c_{3}=\frac{q q_{1}}{\left(q_{1}-q_{2}\right)\left(q-q_{2}\right)},
\end{aligned}
$$

where $q_{1}$ and $q_{2}$ are the conjugates of $q$. 
Consider the case when $b_{2} \theta+b_{3} \bar{\theta}=c_{2} \theta+c_{3} \bar{\theta}$. We use the values for $a_{2}, a_{3}, b_{2}, b_{3}, c_{2}$ and $c_{3}$ as given in equations (4) through (9)). Solving for $\left(b_{2} \theta+b_{3} \bar{\theta}\right)^{2}$ (via Maple [6]) given that $b_{2} \theta+b_{3} \bar{\theta}=c_{2} \theta+c_{3} \bar{\theta}$ with $|\theta|=1$ yields

$$
\left(b_{2} \theta+b_{3} \bar{\theta}\right)^{2}=\frac{q^{4}}{\left(q_{1} q+q+q_{1}\right)\left(q_{2} q+q+q_{2}\right)\left(q-q_{1}\right)\left(q-q_{2}\right)}=C_{1}(q) .
$$

Further, for this $\theta$, we get

$$
A_{1}(q):=\left(a_{2} \theta+a_{3} \bar{\theta}\right)^{2}=\frac{(q+1)^{2}}{\left(q_{1} q+q+q_{1}\right)\left(q_{2} q+q+q_{2}\right)\left(q-q_{1}\right)\left(q-q_{2}\right)} .
$$

From

$$
\frac{A_{1}(q)}{C_{1}(q)}=\frac{(q+1)^{2}}{q^{4}}
$$

we see that for $q>\tau$ that $C_{1}(q)>A_{1}$ and hence $C_{1}(q) \geq U(q)$.

Now consider the case when $b_{2} \theta+b_{3} \bar{\theta}=-c_{2} \theta-c_{3} \bar{\theta}$. We use the values for $a_{2}, a_{3}, b_{2}, b_{3}, c_{2}$ and $c_{3}$ as given in equations (4) through (9). Solving for $\left(b_{2} \theta+b_{3} \bar{\theta}\right)^{2}$ (again via Maple [6]) given that $b_{2} \theta+b_{3} \bar{\theta}=-c_{2} \theta-c_{3} \bar{\theta}$ where $|\theta|=1$ yields

$$
\left(b_{2} \theta+b_{3} \bar{\theta}\right)^{2}=\frac{q^{4}}{\left(q_{1} q-q-q_{1}\right)\left(q_{2} q-q-q_{2}\right)\left(q-q_{1}\right)\left(q-q_{2}\right)}=C_{2}(q) .
$$

Further, for this $\theta$, we get

$$
A_{2}(q):=\left(a_{2} \theta+a_{3} \bar{\theta}\right)^{2}=\frac{(q-1)^{2}}{\left(q_{1} q-q-q_{1}\right)\left(q_{2} q-q-q_{2}\right)\left(q-q_{1}\right)\left(q-q_{2}\right)} .
$$

On noticing that

$$
\frac{A_{2}(q)}{C_{2}(q)}=\frac{(q-1)^{2}}{q^{4}}
$$

we see that $C_{2}(q)>A_{2}(q)$ for $q>1$ and hence $C_{2}(q) \geq U(q)$.

So for $q>\tau$ we have $C_{1}(q) \geq U(q)$ and $C_{2}(q) \geq U(q)$; hence

$$
U(q) \leq \min \left(C_{1}(q), C_{2}(q)\right)=C(q) .
$$

For $q<\tau$ there are only two cases to consider. If $q$ is the first Pisot number, where $q^{3}-q-1=0$, then

$$
U(q)=C(q)=\frac{q^{4}}{\left(q_{1} q+q+q_{1}\right)\left(q_{2} q+q+q_{2}\right)\left(q-q_{1}\right)\left(q-q_{2}\right)}=0.3003453559 .
$$

If $q$ is the second Pisot number in $\mathcal{P}$, where $q^{3}-q^{2}-1=0$, then

$$
U(q)=C(q)=\frac{q^{2}}{\left(q_{1} q+1\right)\left(q_{2} q+1\right)\left(q-q_{1}\right)\left(q-q_{2}\right)}=0.3429099932 .
$$

Thus we see that $U(q) \leq C(q)$ and the result follows.

\section{Proof of A LOWER Bound to ALl QUAdRATics}

This section shows that the sequence of $P_{n}$ described in Section 2 is the best possible.

Theorem 3.1. If $q \in \mathcal{P}$ and $\epsilon>0$, then for all but finitely many integer quadratics $P(x)$, we have $|P(q)| \geq \frac{C(q)-\epsilon}{H(P)^{2}}$, where $H$ is the height function, and $C(q)$ is given in Definition 3 . 
Proof. Let $P$ be an integer quadratic, $q \in \mathcal{P}$, and $q_{1}$ and $q_{2}=\bar{q}_{1}$ the two conjugates of $q$. Since $P$ is quadratic and $q$ is of degree three, we know that $P(q) \neq 0$. This implies that $\left|P(q) P\left(q_{1}\right) P\left(q_{2}\right)\right| \geq 1$ and hence

$$
|P(q)| \geq\left|\frac{1}{P\left(q_{1}\right) P\left(q_{2}\right)}\right| .
$$

Since $P\left(q_{1}\right)=\overline{P\left(q_{2}\right)}$ we see that $P\left(q_{1}\right) P\left(q_{2}\right)$ is real and greater than 0 , hence

$$
|P(q)| \geq \frac{1}{P\left(q_{1}\right) P\left(q_{2}\right)} .
$$

We know from Theorem 2.1 that we can find quadratics that evaluate arbitrarily close to zero at $q$. Thus, except for finitely many exceptions,

$$
|P(q)| \geq \frac{1-\epsilon_{0}}{H(P)^{2} \max \left(S\left(q_{1}\right) S\left(q_{2}\right): H(S) \leq 1, S(q)=0\right)}
$$

where $S$ is a real quadratic.

Finding the maximum in equation (10) is the problem of finding a maximum magnitude on a convex polytope; thus the maximal value is attained on an extreme point (i.e., a corner point). We examine the equation

$$
\max \left(S\left(q_{1}\right) S\left(q_{2}\right): H(S) \leq 1, S(q)=0, S\right. \text { is an extreme point) }
$$

more carefully. We see that $S$ is an extreme point if and only if it is of the form $S(x)=a x^{2}+b x+c$ where:

(1) at least two of $a, b$ and $c$ are \pm 1 ,

(2) $S(q)=0$,

(3) $-1 \leq a, b, c \leq 1$.

We can eliminate half of the cases right away by the symmetry $|S(q)|=|-S(q)|$. We examine each of the possible cases for $a, b$ and $c$, when at least two of the three are of modulus 1.

Case 1: Let $S(x)=x^{2}+x+c$. By requirement (2) we have that $c=-q^{2}-q$. But $-q^{2}-q<-1$ which violates requirement (3). Hence this is not a possible value for $S(x)$.

Case 2: Let $S(x)=x^{2}-x+c$. By requirement (2) we have that $c=-q^{2}+q$. By requirement (3) we have that $-1 \leq-q^{2}+q \leq 1$. Thus we have that $q \leq \tau$. Hence, for $q>\tau$, this is not a possible value for $S(x)$.

Case 3: Let $S(x)=x^{2}+b x+1$. By requirement (2) we have that $b=-q-\frac{1}{q}$. But we see that $-q-\frac{1}{q}<-1$ which violates requirement (3). Hence this is not a possible value for $S(x)$.

Case 4: Let $S(x)=x^{2}+b x-1$. By requirement (2) we have that $b=-q+\frac{1}{q}$. By requirement (3) we have that $-1 \leq-q+\frac{1}{q} \leq 1$. Thus we have that $q \leq \tau$. Hence, for $q>\tau$, this is not a possible value for $S(x)$.

Case 5: Let $S(x)=a x^{2}+x+1$. By requirement (2) we have that $a=-\frac{1}{q}-\frac{1}{q^{2}}$. By requirement (3) we have that $-1 \leq-\frac{1}{q}-\frac{1}{q^{2}} \leq 1$. Thus we have that $q \geq \tau$. Hence, for $q<\tau$, this is not a possible value for $S(x)$.

Case 6: Let $S(x)=a x^{2}+x-1$. By requirement (2) we have that $a=-\frac{1}{q}+\frac{1}{q^{2}}$. By requirement (3) we have that $-1 \leq-\frac{1}{q}+\frac{1}{q^{2}} \leq 1$. This is always true if $q>1$. 
Summarizing these 6 cases shows that if $q>\tau$ equation (11) is equal to

$$
\max \left(\left|\left(-\frac{1}{q}+\frac{1}{q^{2}}\right) q_{1}^{2}+q_{1}-1\right|^{2},\left|\left(-\frac{1}{q}-\frac{1}{q^{2}}\right) q_{1}^{2}+q_{1}+1\right|^{2}\right)
$$

and if $q \leq \tau$, then equation (11) is equal to

$$
\begin{array}{r}
\max \left(\left|\left(-\frac{1}{q}+\frac{1}{q^{2}}\right) q_{1}^{2}+q_{1}-1\right|^{2},\left|q_{1}^{2}+\left(-q+\frac{1}{q}\right) q_{1}-1\right|^{2}\right. \\
\left.\left|q_{1}^{2}-q_{1}+\left(-q^{2}+q\right)\right|^{2}\right) .
\end{array}
$$

Or equivalently, taking $\epsilon_{0}=\frac{\epsilon}{C(q)}$ if $q>\tau$, then

$$
\begin{aligned}
|P(q)| & \geq \frac{1-\epsilon_{0}}{H(P)^{2} \max \left(S\left(q_{1}\right) S\left(q_{2}\right): H(S) \leq 1, S(q)=0\right)} \\
& =\frac{1-\epsilon_{0}}{H(P)^{2} \max \left(\left|\left(-\frac{1}{q}+\frac{1}{q^{2}}\right) q_{1}^{2}+q_{1}-1\right|^{2},\left|\left(-\frac{1}{q}-\frac{1}{q^{2}}\right) q_{1}^{2}+q_{1}+1\right|^{2}\right)} \\
& =\frac{1-\epsilon_{0}}{H(P)^{2}} \min \left(\frac{q^{4}}{\left(q_{1} q-q-q_{1}\right)\left(q_{2} q-q-q_{2}\right)\left(q-q_{1}\right)\left(q-q_{2}\right)},\right. \\
& =\frac{C(q)-\epsilon}{H(P)^{2}}
\end{aligned}
$$

and if $q \leq \tau$, then

$$
\begin{aligned}
&|P(q)| \geq \frac{1-\epsilon_{0}}{H(P)^{2} \max \left(S\left(q_{1}\right) S\left(q_{2}\right): H(S) \leq 1, S(q)=0\right)} \\
&=\frac{1-\epsilon_{0}}{H(P)^{2} \max \left(\left|\left(-\frac{1}{q}+\frac{1}{q^{2}}\right) q_{1}^{2}+q_{1}-1\right|^{2},\left|q_{1}^{2}+\left(-q+\frac{1}{q}\right) q_{1}-1\right|^{2},\left|q_{1}^{2}-q_{1}+\left(-q^{2}+q\right)\right|^{2}\right)} \\
&=\frac{1-\epsilon_{0}}{H(P)^{2}} \min \left(\frac{q^{4}}{\left(q_{1} q-q-q_{1}\right)\left(q_{2} q-q-q_{2}\right)\left(q-q_{1}\right)\left(q-q_{2}\right)},\right. \\
&=\left.\frac{q^{2}}{\left(q_{1} q+1\right)\left(q_{2} q+1\right)\left(q-q_{1}\right)\left(q-q_{2}\right)}, \frac{1}{\left(q+q_{1}-1\right)\left(q+q_{2}-1\right)\left(q-q_{1}\right)\left(q-q_{2}\right)}\right) \\
& H(P)^{2}
\end{aligned}
$$

Thus

$$
|P(q)| \geq \frac{C(q)-\epsilon}{H(P)^{2}}
$$

for all but finitely many integer quadratics $P$, which is the desired result.

It is worth pointing out that when $q>\tau$, both of the cases of $C(q)=C_{1}(q)$ and $C(q)=C_{2}(q)$ do occur, as demonstrated in Table 1. This table lists all $q \in \mathcal{P}, 1<$ $q<5$, along with their minimal polynomials, and $C(q)$, and whether $C(q)=C_{2}(q)$. These Pisot numbers are found using the techniques of David Boyd, as described in [4]. The next section discusses when each case occurs, as well as showing that $C(q)<1$. 
TABLE 1. Some calculations of $C(q)$ for various $q$

\begin{tabular}{||l|l|l|c||}
\hline Pisot polynomial & $q$ & $C(q)$ & $C(q)=C_{2}(q) ?$ \\
\hline$x^{3}-x-1$ & 1.324717957 & 0.3003453557 & \\
$x^{3}-x^{2}-1$ & 1.465571232 & 0.3429099932 & \\
$x^{3}-2 x^{2}+x-1$ & 1.754877666 & 0.3429800030 & \\
$x^{3}-x^{2}-x-1$ & 1.839286755 & 0.4133318671 & $\times$ \\
$x^{3}-x^{2}-2 x-1$ & 2.147899036 & 0.3501354747 & $\times$ \\
$x^{3}-2 x^{2}-1$ & 2.205569430 & 0.5080747995 & \\
$x^{3}-3 x^{2}+2 x-1$ & 2.324717957 & 0.4453345199 & \\
$x^{3}-2 x^{2}-x-1$ & 2.546818277 & 0.5309353500 & $\times$ \\
$x^{3}-3 x^{2}+x-1$ & 2.769292354 & 0.5232405572 & \\
$x^{3}-2 x^{2}-2 x-1$ & 2.831177207 & 0.4435746902 & $\times$ \\
$x^{3}-2 x^{2}-3 x-1$ & 3.079595623 & 0.3847011683 & $\times$ \\
$x^{3}-3 x^{2}-1$ & 3.103803403 & 0.6573022517 & \\
$x^{3}-4 x^{2}+3 x-1$ & 3.147899036 & 0.4911183769 & \\
$x^{3}-3 x^{2}-x-1$ & 3.382975768 & 0.6198187695 & $\times$ \\
$x^{3}-4 x^{2}+2 x-1$ & 3.511547142 & 0.5392925792 & \\
$x^{3}-3 x^{2}-2 x-1$ & 3.627365085 & 0.5238048354 & $\times$ \\
$x^{3}-4 x^{2}+x-1$ & 3.806300717 & 0.6215030654 & \\
$x^{3}-3 x^{2}-3 x-1$ & 3.847322102 & 0.4565756315 & $\times$ \\
$x^{3}-4 x^{2}-1$ & 4.060647028 & 0.7429688162 & \\
$x^{3}-5 x^{2}+4 x-1$ & 4.079595623 & 0.5016713072 & \\
$x^{3}-4 x^{2}-x-1$ & 4.287625262 & 0.6821435669 & $\times$ \\
$x^{3}-5 x^{2}+3 x-1$ & 4.365230013 & 0.5427050340 & \\
$x^{3}-4 x^{2}-2 x-1$ & 4.494492837 & 0.5872601546 & $\times$ \\
$x^{3}-5 x^{2}+2 x-1$ & 4.613470268 & 0.6026317629 & \\
$x^{3}-4 x^{2}-3 x-1$ & 4.685779526 & 0.5175073389 & $\times$ \\
$x^{3}-5 x^{2}+x-1$ & 4.835975919 & 0.6842306666 & \\
$x^{3}-4 x^{2}-4 x-1$ & 4.864536512 & 0.4639992754 & $\times$ \\
\hline
\end{tabular}

\section{The FaCT that $C(q)<1$}

We make the observation that $C(q)<1$ for all $q \in \mathcal{P}, 1<q<5$ from Table 1 This section proves that this observation is true in general, for all $q \in \mathcal{P}$.

Theorem 4.1. For all $q \in \mathcal{P}$ we have that $C(q)<1$, and, moreover, $C(q) \rightarrow 1$ as $q \rightarrow \infty$.

Proof. There are only two cases of $q \in \mathcal{P}$ where $q<\tau$, which we compute explicitly. For the first Pisot number, satisfying $q^{3}-q-1, C(q)=0.3003453559<1$. For the second Pisot number, satisfying $q^{3}-q^{2}-1, C(q)=0.3429099932<1$.

So, without loss of generality, we assume that $q>\tau$. Thus we have that

$$
\begin{aligned}
C(q)= & \min \left(\frac{q^{4}}{\left(q_{1} q+q+q_{1}\right)\left(q_{2} q+q+q_{2}\right)\left(q-q_{1}\right)\left(q-q_{2}\right)},\right. \\
& \left.\frac{q^{4}}{\left(q_{1} q-q-q_{1}\right)\left(q_{2} q-q-q_{2}\right)\left(q-q_{1}\right)\left(q-q_{2}\right)}\right) \\
= & \min \left(C_{1}(q), C_{2}(q)\right) .
\end{aligned}
$$


Let us consider $T(q)=\frac{1}{C(q)}$. Write $q_{1}$ as $x+y i, q_{2}$ as $x-y i$ and $q$ as $\frac{1}{q_{1} q_{2}}=\frac{1}{x^{2}+y^{2}}$. Simplifying $T(q)$ we get

$$
\begin{aligned}
T(q)= & \max \left(x^{12}+6 x^{10} y^{2}+15 x^{8} y^{4}+20 x^{6} y^{6}+15 x^{4} y^{8}+6 x^{2} y^{10}\right. \\
& +y^{12}+2 x^{10}+10 x^{8} y^{2}+20 x^{6} y^{4}+20 x^{4} y^{6}+10 x^{2} y^{8}+2 y^{10} \\
& +x^{8}+4 x^{6} y^{2}+6 x^{4} y^{4}+4 x^{2} y^{6}+y^{8}-2 x^{7}-6 x^{5} y^{2}-6 x^{3} y^{4} \\
& -2 x y^{6}-2 x^{6}-2 x^{4} y^{2}+2 x^{2} y^{4}+2 y^{6}-2 x^{5}-4 x^{3} y^{2}-2 x y^{4} \\
& -2 x^{4}+2 y^{4}+x^{2}+y^{2}+2 x+1 \\
& x^{12}+6 x^{10} y^{2}+15 x^{8} y^{4}+20 x^{6} y^{6}+15 x^{4} y^{8}+6 x^{2} y^{10}+y^{12} \\
& -2 x^{10}-10 x^{8} y^{2}-20 x^{6} y^{4}-20 x^{4} y^{6}-10 x^{2} y^{8}-2 y^{10}+x^{8} \\
& +4 x^{6} y^{2}+6 x^{4} y^{4}+4 x^{2} y^{6}+y^{8}+2 x^{7}+6 x^{5} y^{2}+6 x^{3} y^{4}+2 x y^{6} \\
& -2 x^{6}-2 x^{4} y^{2}+2 x^{2} y^{4}+2 y^{6}-2 x^{5}-4 x^{3} y^{2}-2 x y^{4}+2 x^{4} \\
& \left.-2 y^{4}+x^{2}+y^{2}-2 x+1\right) .
\end{aligned}
$$

For convenience, label the first polynomial as $T_{1}=\frac{1}{C_{1}}$ and the second polynomial as $T_{2}=\frac{1}{C_{2}}$. As the unproven cases are for $q>\tau$, we may assume that $-\sqrt{\frac{1}{\tau}} \leq x \leq \sqrt{\frac{1}{\tau}}$ and $-\sqrt{\frac{1}{\tau}} \leq y \leq \sqrt{\frac{1}{\tau}}$.

Consider $T_{1}$ on the region $0 \leq x \leq \sqrt{\frac{1}{\tau}}$ and $-\sqrt{\frac{1}{\tau}} \leq y \leq \sqrt{\frac{1}{\tau}}$. On the line $x=\sqrt{\frac{1}{\tau}}$, there is a local minimum of approximately 1.364498234 at $y=0$. On the line $y=-\sqrt{\frac{1}{\tau}}$, there are no local minimums or maximums, but the boundary value of $x=0$ gives approximately 3.236067980, and $x=\sqrt{\frac{1}{\tau}}$ gives approximately 17.70723545. The line $y=\sqrt{\frac{1}{\tau}}$ is symmetric to the line $y=-\sqrt{\frac{1}{\tau}}$. On the line $x=0$, there is only one local minimum at $y=0$, giving the value of 1 . On the interior of this region there are no local minimums or maximums (there is a saddle point at $y=0, x=0.5550360821$ of approximately 2.047461577). Thus, $T_{1}$ is always greater than or equal to 1 on this region, and is equal to 1 only at $x=y=0$. (See Figure1.)

Consider $T_{2}$ on the region $-\sqrt{\frac{1}{\tau}} \leq x \leq 0$ and $-\sqrt{\frac{1}{\tau}} \leq y \leq \sqrt{\frac{1}{\tau}}$. On the line $x=-\sqrt{\frac{1}{\tau}}$, there is a local minimum of approximately 3.732814929 at $y=0$. On the line $y=-\sqrt{\frac{1}{\tau}}$, there are no local minimums or maximums (in the correct range), but the boundary value of $x=0$ gives approximately 1.347524158 , and $x=-\sqrt{\frac{1}{\tau}}$ gives approximately 3.371362832. The line $y=\sqrt{\frac{1}{\tau}}$ is symmetric to the line $y=-\sqrt{\frac{1}{\tau}}$. On the line $x=0$, there is only one local minimum at $y=0$, giving the value of 1 . On the interior of this region there are no local minimums or maximums. Thus, $T_{2}$ is always greater than or equal to 1 on this region, and is equal to 1 only at $x=y=0$. (See Figure 2.)

Thus $T_{1}$ and $T_{2}$ are strictly greater than 1 on their respective regions, except at the point $x=y=0$. Thus $T(q)>1$ as we cannot have both $x$ and $y$ equal to 0 . 


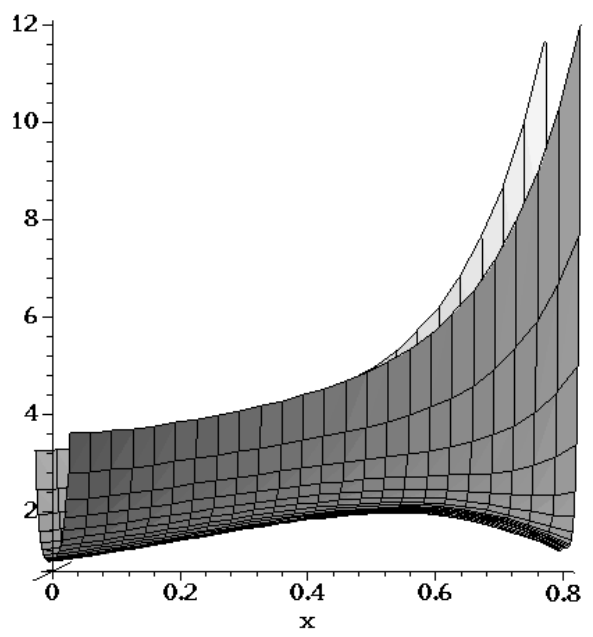

Figure 1. A plot of $T_{1}$ on the region of $x \geq 0$.

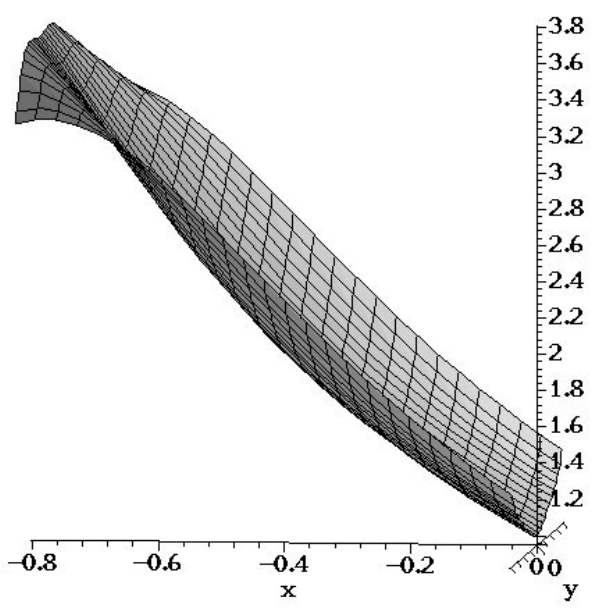

Figure 2. A plot of $T_{2}$ on the region of $x \leq 0$.

We see that if $q \rightarrow \infty$, then $q_{1} \rightarrow 0$ which implies that $x \rightarrow 0$ and $y \rightarrow 0$, thus $T_{1}(x, y) \rightarrow 1$ and $T_{2}(x, y) \rightarrow 1$, and hence $T(q) \rightarrow 1$.

Thus $C(q)<1$ and further as $q \rightarrow \infty$ we have $C(q) \rightarrow 1$.

In Table1 we indicate when $C(q)=C_{2}(q)$. (When $C(q) \neq C_{2}(q)$ and $q>\tau$, then $C(q)=C_{1}(q)$.) Figure 3 gives a graphical interpretation of when $C(q)=C_{2}(q)$. Which value $C(q)$ takes depends on the location of $q_{1}$ in the complex plane. If $q_{1}$ is in the interior of the convex curve (where the real part is less than zero), then $C(q)=C_{1}(q)$, otherwise $C(q)=C_{2}(q)$. 


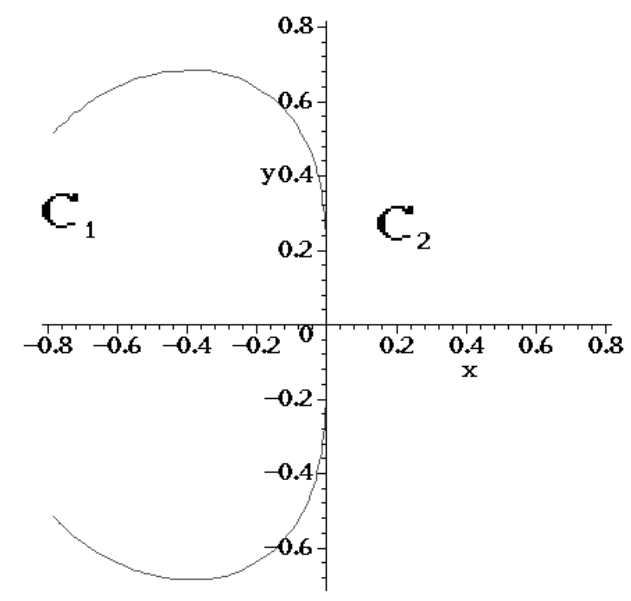

Figure 3 . Regions where $C_{1}$ and $C_{2}$ respectively are minimal.

\section{Bounds FOR THE Height With RESPECT TO $\epsilon$}

Let $q \in \mathcal{P}$. We know from 12 that

$$
|P(q)| \leq \frac{1}{H(P)^{2+\epsilon}}
$$

has only finitely many integer quadratic solutions. Here we find an upper bound for $H(p)$ (dependent on $\epsilon$ ) for all of these solutions.

We see from Theorem 3.1 that $|P(q)| \geq \frac{C(q)-\epsilon}{H(P)^{2}}$ in all but finitely many cases. Let us re-examine the proof of Theorem 3.1 to find a lower bound for $|P(q)|$ that holds in all cases. We rewrite equation (10) as

$$
|P(q)| \geq \frac{1}{H(P)^{2} \max \left(S\left(q_{1}\right) S\left(q_{2}\right): H(S) \leq 1, \operatorname{deg}(S) \leq 2\right)}
$$

by not binding $S(q)$ arbitrarily close to zero. This is true for all integer quadratics $P$. Again, we notice $S$ must be an extreme point, hence this can be rewritten as

$$
|P(q)| \geq \frac{1}{H(P)^{2} \max \left(S\left(q_{1}\right) S\left(q_{2}\right): S(x)= \pm x^{2} \pm x \pm 1\right)} .
$$

So we see that there is a solution only if

$$
\begin{aligned}
\frac{1}{H(P)^{2+\epsilon}} & \geq P(q), \\
\frac{1}{H(P)^{2+\epsilon}} & \geq \frac{q}{H(P)^{2} \max \left(S\left(q_{1}\right) S\left(q_{2}\right): S(x)= \pm x^{2}+ \pm x+ \pm 1\right)} \\
\frac{1}{H(P)^{\epsilon}} & \geq \frac{1}{\max \left(S\left(q_{1}\right) S\left(q_{2}\right): S(x)= \pm x^{2}+ \pm x+ \pm 1\right)} \\
H(P)^{\epsilon} & \leq \max \left(S\left(q_{1}\right) S\left(q_{2}\right): S(x)= \pm x^{2}+ \pm x+ \pm 1\right) \\
H(P) & \leq\left(\max \left(S\left(q_{1}\right) S\left(q_{2}\right): S(x)= \pm x^{2}+ \pm x+ \pm 1\right)\right)^{1 / \epsilon} .
\end{aligned}
$$


This proves the following theorem.

Theorem 5.1. If $q \in \mathcal{P}$ with $q_{1}=\bar{q}_{2}$ the conjugates of $q$ and

$$
H(P)>\left(\max \left(S\left(q_{2}\right) S\left(q_{1}\right): S(x)= \pm x^{2} \pm x \pm 1\right)\right)^{1 / \epsilon},
$$

then there are no integer quadratic solutions $P$ to

$$
|P(q)| \leq \frac{1}{H(P)^{2+\epsilon}} .
$$

\section{Further RESEARCH}

This paper is complete in itself, but it doesn't give a proper accounting on where this problem occurs, and why we are looking at it in the first place. For this we need one more definition.

Definition 4. Define $l^{m}(q)=\inf (|p(q)|: p(q) \neq 0, H(p) \leq m)$.

There is much research on $l^{m}(q)$ when $q$ is Pisot. For an overview, see [2, 8]. It is conjectured, but not known that $l^{1}(q)>0$ if and only if $q$ is Pisot. For general $m$ and $q$ the Golden ratio, $l^{m}(q)$ is known. If $F_{k}$ is the $k$ th Fibonacci number $\left(F_{0}=\right.$ $\left.0, F_{1}=1, F_{n}=F_{n-1}+F_{n-2}\right)$, and $q^{k-2}<m \leq q^{k-1}$, then $l^{m}(q)=\left|F_{k} q-F_{k+1}\right|$ 9]. In 2 a method to calculate $l^{m}(q)$ for all Pisot numbers $q$ and all integers $m$ is given. A tabulation of other $l^{m}(q)$ for various $m$ and $q$, based on these methods of calculations is found at 7]. Upon examination of these tables, we find other patterns similar to that of $l^{m}(q)$ for $q$ is the Golden ratio. One such pattern is described in [3] for all unit quadratic Pisot numbers. Another pattern is for the first and second Pisot numbers, $\omega_{1}^{3}-\omega_{1}-1=0$ and $\omega_{2}^{3}-\omega_{2}^{2}-1=0$. It appears that $l^{m}\left(\omega_{1}\right)=P_{n}\left(\omega_{1}\right)$ for some $n$ dependent on $m$ and $l^{m}\left(\omega_{2}\right)=P_{k}\left(\omega_{2}\right)$ for some $k$ dependent on $m$. This is verified up to $m=39$ for the first Pisot number and up to $m=47$ for the second Pisot number. Both $\omega_{1}$ and $\omega_{2}$ are members of $\mathcal{P}$.

It appears that something also happens for the Pisot root of the polynomial $x^{4}-x^{3}-1$. Even less work is done for this case to determine if the pattern continues, or if these ideas can be extended to an even wider class of Pisot numbers.

This paper leaves some questions unanswered.

(1) Is this pattern for the first two cubic Pisot numbers in fact true for all $m$ ? (This we believe is true.)

(2) If so, what is the relationship between $n$ and $m$ in the equation $P_{n}\left(\omega_{i}\right)=$ $l^{m}\left(\omega_{i}\right)$, for $i=1,2$ ?

(3) Is this pattern true to a limited extent for any of the other Pisot numbers $q$ in $\mathcal{P}$ ? (Which we also believe to be the case.)

If we define $C_{\max }(q):=\max _{|\theta|=1}\left(\max \left(\left|a_{2} \theta+a_{3} \bar{\theta}\right|,\left|b_{2} \theta+b_{3} \bar{\theta}\right|,\left|c_{2} \theta+c_{3} \bar{\theta}\right|\right)\right)^{2}$, then we believe that:

Conjecture 1. For $q \in \mathcal{P}$, for all $m, l^{m}(q)=P_{n}(q)$ for some $n$ if and only if $C_{\max }(q)<1$.

However we also believe that

Conjecture 2. For $q \in \mathcal{P}, C_{\max }(q)<1$ if and only if $q$ is the first or second Pisot number.

Finally, we believe that

Conjecture 3. For $q \in \mathcal{P}$, for infinitely many $n, P_{n}(q)=l^{m}(q)$ for some $m$. 


\section{REFERENCES}

1. J. M. Borwein and P. B. Borwein, Pi and the AGM, John Wiley \& Sons Inc., New York, 1998, A study in analytic number theory and computational complexity, Reprint of the 1987 original, A Wiley-Interscience Publication. MR 99h:11147

2. Peter Borwein and Kevin G. Hare, Some computations on the spectra of Pisot and Salem numbers, Math. Comp. 71 (2002), no. 238, 767-780 (electronic). MR 2003a:11135

3. - General forms for minimal spectral values for a class of quadratic Pisot numbers, Bull. London Math. Soc. 35 (2003), no. 1, 47-54. MR 2003i:11154

4. David W. Boyd, Pisot and Salem numbers in intervals of the real line, Math. Comp. 32 (1978), no. 144, 1244-1260. MR 58:10812

5. J. W. S. Cassels, An introduction to Diophantine approximation, Cambridge University Press, New York, 1957, Cambridge Tracts in Mathematics and Mathematical Physics, No. 45. MR 19:396h

6. K.O. Geddes, G. Labahn, M. B. Monagan, and S. Vorketter, The maple programming guide, Springer-Verlag, New York, 1996.

7. K. G. Hare, Home page, http://www.cecm.sfu.ca/ kghare, 1999.

8. I. Joó and F. J. Schnitzer, On some problems concerning expansions by noninteger bases, Anz. Österreich. Akad. Wiss. Math.-Natur. Kl. 133 (1996), 3-10 (1997). MR 99b:11008

9. V. Komornik, P. Loreti, and M. Pedicini, An approximation property of Pisot numbers, J. Number Theory 80 (2000), no. 2, 218-237. MR 2000k:11116

10. Maurice Mignotte, Mathematics for computer algebra, Springer-Verlag, New York, 1992, Translated from the French by Catherine Mignotte. MR 92i:68071

11. K. F. Roth, Rational approximations to algebraic numbers, Mathematika 2 (1955), 1-20; corrigendum, 168. MR 17:242d

12. Wolfgang M. Schmidt, On simultaneous approximations of two algebraic numbers by rationals, Acta Math. 119 (1967), 27-50. MR 36:6357

Department of Mathematics and Statistics, Simon Fraser University, Burnaby, British Columbia, Canada V5A $1 \mathrm{~S} 6$

E-mail address: pborwein@cecm.math.sfu.ca

Department of Mathematics and Statistics, Simon Fraser University, Burnaby, BriTish Columbia, Canada V5A 1 S6

E-mail address: kghare@cecm.math.sfu.ca 